ERRAT UM

M. Tsokos $\cdot$ S. Anders $\cdot$ F. Paulsen

\title{
Lectin binding patterns of alveolar epithelium and subepithelial seromucous glands of the bronchi in sepsis and controls - an approach to characterize the non-specific immunological response of the human lung to sepsis
}

Published online: 30 May 2002

(C) Springer-Verlag 2002

\section{Virchows Arch (2002) 440:181-186}

Due to a printer's error, the first two columns of the following two tables were misprinted in the print version of this contribution. Here are the two tables, Tables 3 and 4, in their correct form.
The online version of the original article can be found at http:// dx.doi.org/10.1007/s004280100488

M. Tsokos ( $)$ S. Anders

Institute of Legal Medicine, University of Hamburg,

Butenfeld 34, 22529 Hamburg, Germany

e-mail: ifrhh@uke.uni-hamburg.de

Tel.: +49-40-42803-2748

Fax: +49-40-42803-3934

S. Anders

Institute of Pathology, University of Hamburg,

Martinistrasse 52, 20246 Hamburg, Germany

F. Paulsen

Department of Anatomy, Christians-Albrechts-University of Kiel,

Olshausenstrasse 40, 24098 Kiel, Germany

Table 3 Summary of lectin binding to alveolar epithelial cells in sepsis and controls

\begin{tabular}{|c|c|c|c|c|c|c|c|c|c|c|c|c|}
\hline Study group & Case number & Con A & $M A A$ & $U E A$ & $J a c$ & $G S A I$ & GSAII & $P N A$ & $M P A$ & SNA & $W G A$ & $L P A$ \\
\hline \multirow[t]{4}{*}{ Sepsis } & 1 & +++ & ++ & - & - & - & - & - & - & - & - & - \\
\hline & 2 & +++ & + & - & - & - & - & - & - & +++ & - & - \\
\hline & 3 & +++ & + & - & ++ & - & - & - & - & +++ & - & - \\
\hline & 4 & +++ & + & - & +++ & - & - & - & - & +++ & - & - \\
\hline \multirow{4}{*}{ Control } & 1 & +++ & - & - & +++ & - & - & - & + & +++ & - & - \\
\hline & 2 & +++ & + & - & - & - & - & - & ++ & - & - & - \\
\hline & 3 & +++ & ++ & - & +++ & - & - & - & + & +++ & - & - \\
\hline & 4 & +++ & - & - & +++ & - & - & - & + & +++ & - & - \\
\hline
\end{tabular}

Table 4 Summary of lectin binding to mucous parts of subepithelial seromucous glands

\begin{tabular}{|c|c|c|c|c|c|c|c|c|c|c|c|c|}
\hline Study group & Case number & $\operatorname{Con} A$ & $M A A$ & $U E A$ & $J a c$ & GSAI & GSAII & $P N A$ & $M P A$ & SNA & $W G A$ & $L P A$ \\
\hline \multirow[t]{3}{*}{ Sepsis } & 1 & - & - & - & +++ & - & - & +++ & - & +++ & ++ & - \\
\hline & 2 & - & - & - & +++ & - & - & - & - & - & ++ & - \\
\hline & 4 & - & - & - & - & - & - & - & - & - & +++ & - \\
\hline \multirow{2}{*}{ Control } & 1 & ++ & - & - & - & - & - & - & ++ & - & +++ & - \\
\hline & 2 & - & - & - & +++ & - & - & - & +++ & +++ & ++ & - \\
\hline
\end{tabular}

